\title{
Myocardial feature tracking for viability assessment in ischemic cardiomyopathy
}

\author{
Andreas Schuster ${ }^{1 *}$, Matthias Paul ${ }^{1}$, Nuno Bettencourt ${ }^{2}$, Tarique Hussain ${ }^{1}$, Dirk Lossnitzer ${ }^{1}$, Masaki Ishida ${ }^{1}$, \\ Amedeo Chiribiri', Boris Bigalke', Geraint Morton ${ }^{1}$, Divaka Perera ${ }^{1}$, Gerald Greil ${ }^{1}$, Eike Nagel ${ }^{1}$ \\ From 2011 SCMR/Euro CMR Joint Scientific Sessions \\ Nice, France. 3-6 February 2011
}

\section{Objective}

To evaluate myocardial feature tracking (FT) as a potential novel quantitative analysis tool for the assessment of myocardial viability using LDDSMR.

\section{Background}

Low dose dobutamine stress magnetic resonance imaging (LDDSMR) is a widely accepted technique for assessment of hibernating myocardium in patients with ischemic cardiomyopathy (ICMP). It is particularly helpful in patients with intermediate transmurality of scar where prediction of functional recovery by late gadolinium enhancement imaging (LGE) alone is difficult. Analysis of LDDSMR is usually based on visual assessment and is therefore considerably operator dependant. Recently myocardial feature tracking (FT) has been introduced. It tracks tissue voxel motion of cine-MR images to assess circumferential and radial myocardial strain independent of additional sequences.

\section{Methods}

15 consecutive patients with ICMP referred for viability assessment were studied at 3 Tesla (Philips Achieva) at rest and during LDDSMR. ICMP was defined as: angiographically established coronary artery disease, LV-EF $\leq 45 \%$ and $\geq 2$ segments with wall motion abnormalities at rest. Myocardial function was studied by steady state free precession (SSFP) cine imaging in 3 short axis slices covering 16 myocardial segments, excluding the apex.

The same segments were studied with dedicated FT software (Diogenes MRI, Tomtec, Germany) to assess

${ }^{1}$ King's College London BHF Centre of Excellence, Division of Imaging Sciences, NIHR Biomedical Research Centre at Guy's and St. Thomas' NHS Trust Foundation, Wellcome Trust and EPSRC Medical Engineering Centre

The Rayne Institute, St. Thomas' Hospital, London, UK

Full list of author information is available at the end of the article subendocardial and subepicardial circumferential (Ecc) and radial (Err) strain.

\section{Results}

208 of 240 segments $(87 \%)$ were analysed by two independent observers. 32 segments were excluded because of insufficient image quality or visualization of the outflow tract. Interobser-variability was: Ecc subendocardial $\mathrm{r}=0.84$, Ecc subepicardial $\mathrm{r}=0.72$ and Err $\mathrm{r}=0.7$.

During LDDSMR ( 5 and $10 \mu \mathrm{g} \cdot \mathrm{kg}^{-1} \cdot \mathrm{min}^{-1}$ ) there was improvement in Ecc subendocardial, Ecc subepicardial $(\mathrm{p}<0.001$, by repeated measures analysis) and Err $(\mathrm{p}=0.001$, please refer also to Table 1$)$. Normokinetic segments without scar $(\mathrm{n}=67)$ improved in Ecc subendocardial $(p<0.001)$ and Ecc subepicardial $(p=0.008)$ while Err remained unchanged with stress. Hypokinetic segments without scar $(n=75)$ improved in all three strain values (Ecc subendocardial $\mathrm{p}=0.012$; Ecc subepicardial $\mathrm{p}=0.035$; Err $\mathrm{p}=0.001)$. Hypokinetic segments with non-transmural scar $(n=60)$ showed improved Ecc subendocardial, Ecc subepicardial and Err up to $75 \%$ transmurality while transmurally scarred segments $(n=6)$ remained unchanged with stress.

\section{Conclusion}

FT is a novel technique, which reliably detects quantitative wall motion derived from SSFP CINE imaging. It seems useful for quantitative analysis at rest and during low dose dobutamine stress for viability assessment in patients with ischemic cardiomyopathy.

\section{Author details}

${ }^{1}$ King's College London BHF Centre of Excellence, Division of Imaging Sciences, NIHR Biomedical Research Centre at Guy's and St. Thomas' NHS Trust Foundation, Wellcome Trust and EPSRC Medical Engineering Centre; The Rayne Institute, St. Thomas' Hospital, London, UK. '2Cardiology Department - Centro Hospitalar de Gaia/Espinho, Porto, Portugal. 
Table 1 Strain Values for different groups of transmurality at rest, 5 and $10 \mu \mathrm{g} \cdot \mathbf{k g}-\mathbf{1} \cdot \mathbf{m i n}-1$ of dobutamine

\begin{tabular}{|c|c|c|}
\hline Strain & Strain Rest, 5 and $10 \mu \mathrm{g} \cdot \mathrm{kg}^{-1} \cdot \mathrm{min}^{-1}$ of dobutamine & $\mathbf{p}$ \\
\hline \multicolumn{3}{|c|}{ All segments (WMS 0-5, LGE 0-100\%, $n=208$ ) } \\
\hline ECC subendocardial & $-11.8 \pm 8.9 ;-14.1 \pm 9.7 ;-16.2 \pm 11.2$ & $<0.001$ \\
\hline EcC subepicardial & $-7.1 \pm 5.2 ;-8.9 \pm 6.1 ;-10.4 \pm 7.8$ & $<0.001$ \\
\hline Err & $13.4 \pm 12 ; 16.1 \pm 12.1 ; 17.2 \pm 14.3$ & 0.001 \\
\hline \multicolumn{3}{|c|}{ Normal segments (WMS 5, LGE 0\%, $\mathrm{n}=67$ ) } \\
\hline ECC subendocardial & $-16.2 \pm 11.1 ;-18.9 \pm 11.5 ;-22.3 \pm 13.3$ & $<0.001$ \\
\hline ECC subepicardial & $-8.6 \pm 6.2 ;-10.6 \pm 7.4 ;-13 \pm 10.3$ & 0.008 \\
\hline Err & $19.3 \pm 15 ; 20.7 \pm 13.2 ; 21.4 \pm 15.7$ & 0.675 \\
\hline \multicolumn{3}{|c|}{ Hypokinetic segments without scar (WMS<5, LGE 0\%, n=75) } \\
\hline ECC subendocardial & $-10.5 \pm 6.9 ;-12.1 \pm 6.9 ;-14.1 \pm 9.2$ & 0.012 \\
\hline EcC subepicardial & $-7 \pm 4.8 ;-8.2 \pm 5.5 ;-9.1 \pm 5.9$ & 0.035 \\
\hline Err & $11.7 \pm 8.3 ; 16 \pm 10.9 ; 16.5 \pm 12.8$ & 0.001 \\
\hline \multicolumn{3}{|c|}{ Hypokinetic segments with non-transmural scar (WMS<5, LGE 1-25\%, n=16) } \\
\hline ECC subendocardial & $-10.7 \pm 7.9 ;-16 \pm 8.7 ;-16.6 \pm 10.8$ & $<0.001$ \\
\hline Ecc subepicardial & $-7.6 \pm 5.3 ;-10.4 \pm 6.6 ;-12.2 \pm 7.5$ & 0.016 \\
\hline Err & $11.7 \pm 7.19 ; 20.1 \pm 16.8,20.3 \pm 18.6$ & 0.075 \\
\hline \multicolumn{3}{|c|}{ Hypokinetic segments with non-transmural scar (WMS<5, LGE 26-75\%, n=44) } \\
\hline Ecc subendocardial & $-7.6 \pm 5.2 ;-10.3 \pm 7.4 ;-11.8 \pm 7.7$ & $<0.001$ \\
\hline EcC subepicardial & $-5.6 \pm 3.8 ;-7.4 \pm 4.3 ;-8.5 \pm 4.9$ & 0.001 \\
\hline Err & $8.4 \pm 10.1 ; 8.7 \pm 5.6 ; 12.3 \pm 12$ & 0.017 \\
\hline \multicolumn{3}{|c|}{ Hypokinetic segments with transmural scar (WMS<5, LGE 76-100\%, n=6) } \\
\hline EcC subendocardial & $-4.7 \pm 3 ;-2.9 \pm 2.5 ;-6.6 \pm 3.3$ & 0.214 \\
\hline EcC subepicardial & $-2.9 \pm 2.9 ;-5.4 \pm 3.9 ;-4.5 \pm 4.2$ & 0.131 \\
\hline Err & $9.51 \pm 5 ; 5.4 \pm 6.2 ; 4.9 \pm 3.3$ & 0.543 \\
\hline
\end{tabular}

Published: 2 February 2011

doi:10.1186/1532-429X-13-S1-P153

Cite this article as: Schuster et al: Myocardial feature tracking for

viability assessment in ischemic cardiomyopathy. Journal of

Cardiovascular Magnetic Resonance 2011 13(Suppl 1):P153.

Submit your next manuscript to BioMed Central and take full advantage of:

- Convenient online submission

- Thorough peer review

- No space constraints or color figure charges

- Immediate publication on acceptance

- Inclusion in PubMed, CAS, Scopus and Google Scholar

- Research which is freely available for redistribution

Submit your manuscript at www.biomedcentral.com/submit
C Biomed Central 